\title{
Approfondir la Méthode de François Delsarte : Les Attitudes du Torse et l'Equilibre Harmonique
}

\author{
Franck Waille \\ Laboratoire de Recherche Historique Rhône-Alpes (LARHRA), France \\ E-mail : franck.cw@gmail.com
}

\section{Résumé}

La reconstruction des enseignements pratiques (pour le corps, et encore plus pour la voix) de François Delsarte relève d'un vrai défit méthodologique : si ces enseignements ont joué un rôle déterminant dans l'émergence de la modernité des arts de la scène, des pratiques psychosomatiques et de l'éducation somatique, ils ont essentiellement été transmis de manière " orale ", de maître à élève. Et ils ont été, pour une part, perdus. C'est à partir des éléments de transmission textuelle, associés à une pratique de la danse moderne, du théâtre et des approches somatiques, qu'il est possible d'en retrouver une compréhension aussi proche que possible de leurs versions d'origine, sans avoir la prétention de pouvoir revenir à celles-ci. D'autant plus que les très nombreuses nuances que Delsarte introduisait dans son enseignement invitent à une dynamique ouverte : non chercher « la lettre » mais « l'esprit » de son travail, dans une perspective de renouvellement constant. Cette démarche est celle de la recherche de Franck Waille, qui ne cesse de s'affiner depuis le début des années 2000. Elle concerne différents éléments de la Méthode somatique expressive de Delsarte. Cet article présente une mise à jour concernant les attitudes du torse, favorisée par la rencontre avec la recherche de Clóvis Massa sur Delsarte faite au début des années 1990.

\section{Palavras-chave}

François Delsarte. Attitudes / Accord de neuvième. Expression des jambes. Arts de la scène. Éducation somatique.
Abstract

The reconstruction of the practical teachings (for the body, and even more the voice) of François Delsarte raises a real methodological challenge : if these teachings played a determining role in the emergence of the modernity of the performing arts, practices psychosomatic and somatic education, they were essentially transmitted "orally" from master to student. And they were, for one, lost. It is from the elements of textual transmission, associated with a practice of modern dance, drama and somatic approaches, that it is possible to find an understanding as close as possible to their original versions, without pretend to be able to return to these. Especially since the many nuances that Del-sarte introduced into his teaching invite an open dynamic : not looking for "the letter" but "the spirit" of his work, in a perspective of constant renewal. This approach is that of the research of Franck Waille, which has been constantly refined since the beginning of the 2000s. It concerns different elements of Delsarte's expressive somatic method. This article presents an update on the attitudes of the torse, favored by the meeting with the research of Clóvis Massa on Delsarte made in the early 1990s.
Keywords

François Delsarte. Attitudes/Ninth Agreement. Expression of the torso. Scenic arts. Somatic education. 


\section{Introduction}

Dans un précédent article $^{1}$, j'ai présenté mon approche renouvelée du travail des attitudes des jambes chez François Delsarte (1811-1871). Je présente ici mon approche renouvelée du travail des attitudes des jambes et de la notion "d'équilibre harmonique » caractéristique des transmissions delsartiennes. L'approche renouvelée des attitudes des jambes a été favorisée, lors de mon second postdoctorat au Département d'Éducation de l'Universidade Federal do Rio Grande do Sul (UFRGS) en 2016 et 2017, par la rencontre avec la recherche de Clóvis Massa faite sur Delsarte au début des années 1990. Cette recherche m'a amené à faire une relecture des manuscrits concernant ce travail des jambes chez Delsarte, travail qui s'inscrit dans le cadre plus général de l'entrainement expressif des différentes parties du corps avec l'outil delsartien appelé l'accord de neuvième, qui a été la source de nombreuses ambiguïtés dans la transmission et la réception des enseignements de Delsarte. J'ai donc, avant de présenter par des textes et des photographies les apports combinés de mes recherches et de celles de Clóvis Massa concernant les attitudes des jambes, fait une synthèse concernant l'accord de neuvième, afin d'en bien saisir les dynamiques.

Les attitudes des jambes sont la base de l'organisation corporelle générale et de la dynamique. Mais les attitudes du torse jouent elles aussi un grand rôle dans l'organisation globale du corps, du fait que le torse soit l'élément central de l'organisme, et parce que ses attitudes interagissent automatiquement sur les positions de la tête et du cou, et donc sur le « contrôle primaire $»^{2}$. Elles ont dès lors un lien direct avec

\section{Approfondir la méthode de François Delsarte : Les attitudes} des jambes.

2 Selon F.M. Alexander, le « contrôle primaire » [primary control] de la coordination du corps se ferait au niveau du cou. L'apport majeur d'Alexander est d'avoir mis en valeur la relation qui existe entre la tête, le cou et le dos : si cette relation est « juste », l'organisme entier peut fonctionner de façon optimale. Autrement dit, en retrouvant l'alignement équilibré et dynamique de ces trois régions, on restaurerait le bon fonctionnement du la notion « d'équilibre harmonique ». Dans ma thèse Corps, arts et spiritualité chez François Delsarte (1811-1871) (2009), je me suis beaucoup appuyé pour le travail de l'équilibre harmonique sur les informations fournies par la delsartiste américaine Genevieve Stebbins (1977) qui ont l'immense avantage d'une très grande clarté (des leçons très organisées et détaillées). Je relevais cependant déjà des écarts par rapport aux manuscrits plus proches des enseignements originaux de Delsarte. Dans mon ouvrage La méthode somatique expressive de François Delsarte (2016), je précisais ces écarts et proposais une réorganisation partielle du travail présenté en 2009, étant alors plus fidèle aux manuscrits delsartiens. Mon premier postdoctorat fait au Département de Danse de I'Université du Québec À Montréal (UQAM) en 2014 et 2015 m'avait au préalable permis de poursuivre l'exploration de manuscrits autour du travail du torse. Depuis, je me suis laissé interroger à nouveau par les différents manuscrits, et les apports de la rencontre avec la recherche de Clóvis Massa sur Delsarte, signalés dans mon précédent article, ont également eu une incidence sur mon approche actuelle du travail du torse.

Le présent article est donc le troisième mo-

corps et on réhabiliterait le réflexe d'antigravité naturel, que l'on peut en général observer chez les petits enfants (cf. Alexander, 1996 [1923], chapitre 1 en particulier). II y a des similitudes avec les recherches, à la même époque, de Georges Coghill sur les salamandres (leur corps s'organise depuis la tête - cf. https:// www.alexander-technique.london/alexander-technique-articles/ primary-control/) et de Rudolf Magnus sur le Zentralapparat (contrôle central, " la tête qui mène " - cf. http://alexander. area24.net/RudolfMa-gnus-AnimalPosture.htm) (https://www. alexander-technique.london/alexander-technique-articles/primary-control/). Alexander a très probablement été influencé par le travail de Delsarte qu'il connaissait et transmettait. D'une part, parce que chez Delsarte la question de l'équilibre et de la verticalité est un rapport entre le torse, la tête et les membres [ou la région du bassin] (nous y revenons dans les pages qui viennent). D'autre part, parce que Delsarte mettait en valeur le rôle de la tête dans les mouvements expressifs : il avait observé que les « inflexions passent (...) alternativement de la tête à la main " (1839. p. 52) et parlait alors de " transmissions " qu'il pouvait également décrire ainsi : "Les gestes passent de la tête à tous les agents du corps " (Mackaye, 1869/1870a : 72). Son expérience de chanteur a très certainement favorisé ces directions pédagogiques concernant la verticalité ainsi que ses observations, puisque dans le chant « l'émission d'un son harmonieux requiert l'équilibre entre la tête et le reste du corps, dans ce lieu rétréci qu'est le cou » (Barthélémy, 1984. p. 39). 
ment d'un ensemble dont les deux premiers éléments (dynamiques de l'accord de neuvième et approche renouvelée des attitudes des jambes) ont été présentés dans un précédent article. Comme pour les jambes, je présente ici mon exposé sur les attitudes du torse et la notion d'équilibre harmonique avec des textes et des images. Je précise à nouveau que les photographies ne prétendent en rien être des versions définitive, absolues ou de référence des exercices auxquels elles se référent. Elles sont de simples illustrations d'une interprétation de ces exercices à une moment de ma recherche (elles proviennent, par ailleurs, de différents séries de prises de vue). Elles ont donc pour seul objectif d'apporter un support visuel au texte, et elles comportent de nombreuses imperfections que le lecteur me pardonnera... et qu'il aura, je l'espère, le goût de gommer en s'impliquant dans la pratique des exercices exposés !

\section{Les attitudes du torse et la notion d'équilibre harmonique}

Parce que le torse est l'élément central du corps, mettant en lien le bassin et la tête dans l'axe vertical, il joue un rôle éminent dans l'organisation générale de l'organisme et dans ses dynamiques expressives. C'est la raison pour laquelle il est forcément associé à la notion d'équilibre harmonique. Mais nous verrons que celle-ci, réduite dans certaines transmissions américaines à cette seule gestion du torse, a en fait une acceptation plus large chez Delsarte, et cela dès les origines de ses enseignements.

Les attitudes du torse ne bénéficient que d'un manuscrit delsartien de 1839, le compterendu des tout premiers cours d'Esthétique Appliquée (c'est ce même manuscrit qui me servira de base pour présenter la vision élargie de l'équilibre harmonique). On peut facilement imaginer que ce travail se soit approfondi durant les trente-deux ans qui séparent ce manuscrit de la fin de la carrière de Delsarte en 1871. Les transmissions variées de ces attitudes par tous ses élèves ayant publié des éléments de son travail pratique rendent probablement compte de ces approfondissements potentiels. Mais nous verrons que le manuscrit de 1839 bénéficie déjà d'un contenu d'une telle richesse qu'aucun des élèves directs de Delsarte n'a su ou voulu en rendre compte en totalité. Si j'ai, dès ma thèse, énoncé cette richesse, montrant comment la plupart des différences de transmission pouvaient trouver des référents dans ce que présentait ce manuscrit, il manquait à ma démonstration la «preuve par l'image ». II se trouve que l'approfondissement du rôle du thorax pour les attitudes des jambes permis par la rencontre avec le travail de Clóvis Massa s'est fait durant la même période de ma recherche où je revenais sur ce manuscrit de 1839 ; il y a donc une dynamique autour du torse dans mon approche renouvelée des enseignements delsartiens.

Dans les photographies qui accompagnent la suite de cet article, je ne présente que les positions finales des exercices (j'en ai présenté des versions séquencées précédemment - cf. Waille, 2011, p. 799-814, \& Waille 2016, p. 381426).

\section{Les attitudes du torse dans le manuscrit de 1839}

Le cahier de 1839, École de Delsarte, École de chant morale et scientifique. Notes et compte-rendu de ses cours [1839] témoigne des deux premiers mois de l'enseignement public de Delsarte dans le cadre des cours d'Esthétique Appliquée donnés à l'attention d'un petit groupe d'élèves (cf. Waille, 2011, p. 232-233). Ce sont ces élèves qui ont réuni dans un même cahier des transcriptions de l'enseignement de leur maître, sous couvert de ce dernier, qui relisait et signait ces documents. Le travail était collectif et s'appuyait sur l'ensemble des notes spontanées des élèves prises in vivo, puis synthétisées. Cette rédaction avait $\AA$ pour but de laisser une trace consultable après les cours, mais surtout d'alimenter le cours suivant, qui s'ouvre par sa lecture. Elle est enrichie éventuellement des « réponses faites aux questions 
ou aux objections » (Delsarte, 1839, p. 1). C'est seulement après ce travail en plusieurs strates que le résultat final est transcrit sur le cahier qui nous est arrivé. Ce processus montre la claire conscience qu'avaient Delsarte et ses élèves de la multiplicité des façons de recevoir un enseignement, et de la nécessité de croiser ces regards différents pour retrouver au mieux ce qu'a voulu dire le maître.

Voici le passage de ce carnet se rapportant aux attitudes du torse. Je présente d'abord la première partie de ce passage :

Le torse nous présente trois aspects et révèle encore à la pensée les trois états déjà signalés : s'il se présente dans sa plus grande dilatation, il est porté en ce cas en avant, c'est l'attitude de l'homme énergique, c'est l'attitude militaire, nous l'appellerons excentrique. Le torse se présente encore sous un aspect plus homogène, moins contentieux, plus affectif, tel qu'il est représenté dans la belle statue d'Antinoüs, nous l'appellerons ici expansif [normal]. Si les épaules se soulèvent et se portent en avant, la poitrine se creusera et nous donnera l'aspect compressif [concentrique] (Delsarte, 1839, p. 52).

Delsarte commence donc par les trois attitudes du torse dans le plan sagittal (vers l'avant et vers l'arrière), qui est le plan le plus immédiat car le plus commun dans l'expérience de la vie quotidienne (il est étonnant que Stebbins (1977, exercices 1-3 du chapitre II, p. 91-93³) débute elle le travail dans le plan frontal : cela n'est ni en cohérence avec les enseignements de Delsarte, ni avec l'expérience motrice humaine puisque le plan sagittal est le premier plan de développement moteur - cf. Bainbridge-Cohen, 2002). Ce sont donc, dans le cadre de l'accord de neuvième, les trois genres. J'ai mis entre crochets le vocabulaire caractéristique de la plupart des cours de Delsarte (concentrique et normal étaient précédemment dénommés compressif et expansif).

3 Nous nous référons ici non au chapitre XII consacré au torse (il est très pauvre), mais au chapitre II « Harmonic Poise of Bearing » (1977 : 91-102), qui contient l'essentiel du travail pratique pour le torse issu de l'accord de neuvième du torse de Delsarte (cf. infra).
Les photographies font ressortir le fait que les trois genres du torse (Figure 1) impliquent chacun une opposition du thorax avec le bassin et la tête (qui ici travaillent en sympathie : ils vont dans la même direction, opposée à celle du torse) :

Figure 1 : Les trois genres (concentrique, normal, excentrique) du torse (interprétation par l'image)
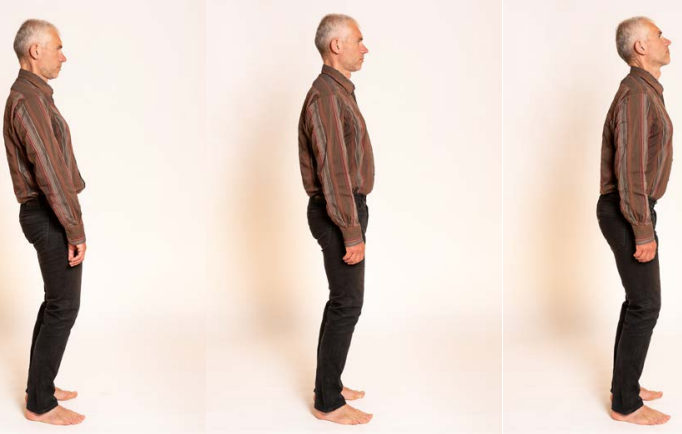

Franck Waille (photographie@David VENIER Université Jean Moulin Lyon 3)

Le travail des trois genres du torse est le plus simple à expérimenter car il n'implique pas (contrairement à celui sur les côtés, dans le plan frontal) de transfert de poids (les oppositions entre le thorax d'une part, la tête et le bassin d'autre part, permettent une stabilité de la répartition du poids au niveau des pieds). De plus, il est aisé d'associer à ce travail l'ajout du rythme respiratoire : sur l'inspiration, le thorax se porte plus volontiers en avant, et sur l'expiration en arrière. Se centrer sur le rythme respiratoire peut aider à entrer dans une intériorité lors du travail et a avoir une prise de conscience fine des processus mis en œuvre en soi à cette occasion, la respiration invitant facilement (« matériellement », par le voyage de l'air en soi) à une attention tournée vers l'intérieur et vers le thorax.

Mackaye indique, pour chacun des genres (Figure 2), l'ajout d'une zone proéminente venant directement de la division esthétique du corps chez Delsarte (bassin vital ; zone épigastrique animique; haut du thorax intellectif) : 
Figure 2 : Genre normal du torse avec proéminence : du bassin/de la zone épigastrique/du haut du thorax (interprétation par l'image)
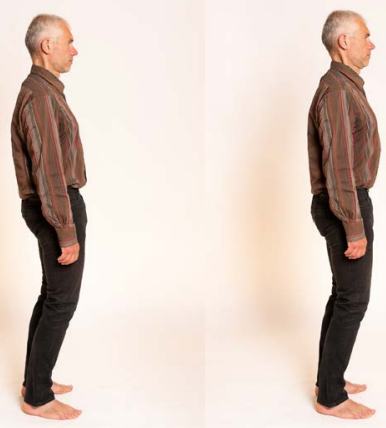

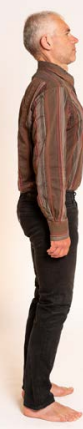

Franck Waille (photographie@David VENIER Université Jean Moulin Lyon 3)

Le travail dans le plan frontal (sur les côtés) est plus complexe du fait des transferts de poids sur une jambe ou sur l'autre indiqués à la fin du texte de 1839, ce qui amène à avoir non pas neuf mais quinze espèces :

Ces trois genres nous donneront encore neuf espèces : ainsi, dans chacun de ces genres, le torse sera penché vers l'interlocuteur, ou contrairement à lui, nous aurons donc trois fois trois et par conséquent le triple accord déjà signalé. Mais ici il se présente des formes additionnelles qui ne s'étaient pas encore présentées : ainsi, nous reconnaîtrons que le torse penché vers l'interlocuteur peut porter dans cette attitude alternativement sur la hanche droite ou gauche. Les six mouvements penchés se doublent donc et nous aurons une somme de quinze mouvements ou quinze espèces (Delsarte, 1839, p. 51-52).

La somme de quinze espèces indique que pour chaque genre, il y a quatre variations (et non pas six), c'est-à-dire qu'à chaque penchement du torse, il y a transfert du poids sur l'une ou l'autre jambe (n'est donc pas envisagée ici l'égale répartition du poids sur les deux jambes). Le genre de départ est le cinquième élément pour aller à quinze espèces : cinq avec le genre normal + cinq avec le genre excentrique + cinq avec le genre concentrique. Voilà, dans le genre normal (Figure 3 et 4), ce que cela peut donner avec un interlocuteur placé à droite (Figure 5) :
Figure 3 : Genre normal de profil

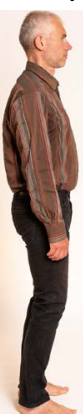

Figure 4 : Genre normal de front

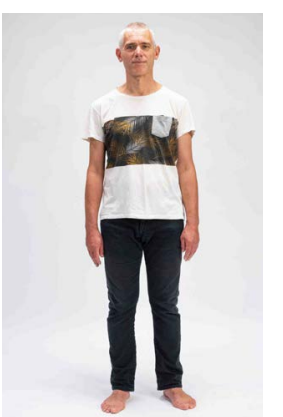

Franck Waille (photographie@David VENIER Université Jean Moulin Lyon 3)

Figure 5 : Attitudes du torse dans le genre normal : quatre espèces avec l'interlocuteur à droite (interprétation par l'image)

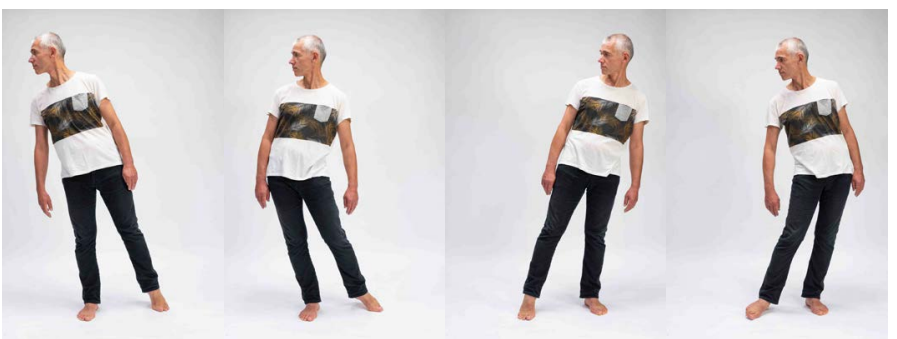

Franck Waille (photographie@David VENIER Université Jean Moulin Lyon 3)

Cet accord avec quinze espèces se double de facto dans la mesure où l'interlocuteur peut être penché à gauche (Figure 6) :

Figure 6 : Attitudes du torse dans le genre normal : quatre espèces avec l'interlocuteur à gauche (interprétation par l'image)

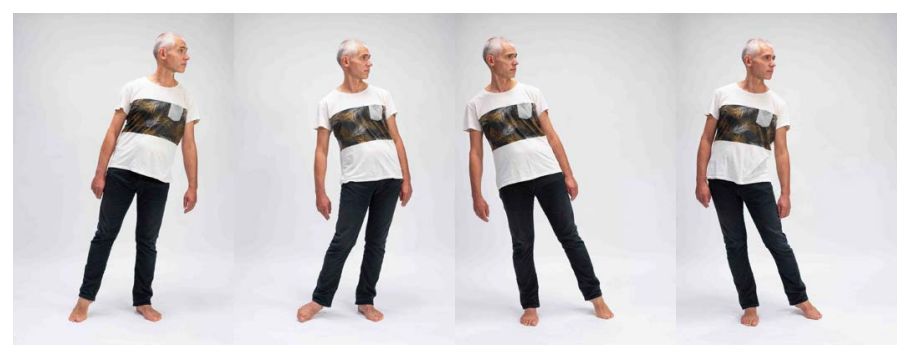

Franck Waille (photographie@David VENIER Université Jean Moulin Lyon 3)

Ces exercices peuvent également être faits assis (Figure 7), puisque cela s'observe dans le quotidien, ce qui correspond au genre normal des jambes (personne assise sur un support) 
que présente Giraudet (1895, p. 87-93) et aux exercices assis transmis pas Stebbins (1977] exercices 10 et 11 en particulier) :

Figure 7 : Attitudes du torse dans le genre normal : quatre espèces en étant assis (interprétation par l'image)

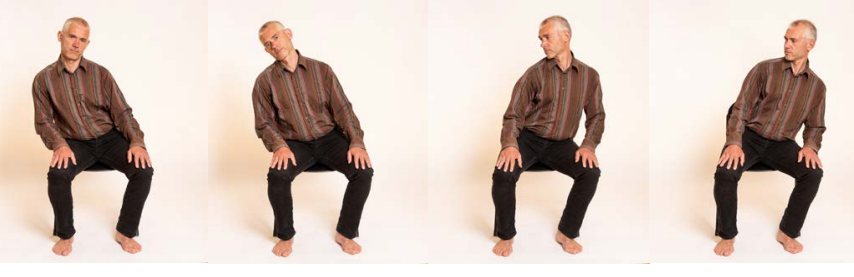

Franck Waille (photographie@David VENIER Université Jean Moulin Lyon 3)

La notion de penchement du torse appelle des remarques:

1) il est clair que le torse peut se pencher dans toutes les directions, et pas uniquement sur les côtés (dans l'axe frontal) : le travail proposé n'est qu'une base permettant d'envisager toutes les possibilités de mouvements du torse en fonction d'un interlocuteur. Dans les transmissions de Giraudet et de Stebbins, il est sans ambiguïté indiqué de travailler dans l'axe frontal (Stebbins ajoute ensuite les diagonales, fidèle en cela à la Médaille Inflective de Delsarte - cf. Waille, 2016, p. 142-146) ;

2) le travail à partir de la recherche de Clóvis Massa m'amène à penser la notion de penchement du torse (que nous avons rencontrée précédemment, en particulier pour la cinquième attitude des jambes) de deux manières : soit en gardant les épaules rigoureusement dans l'axe frontal (comme je le propose dans la position assise), soit en effectuant une légère rotation en direction de l'interlocuteur (comme je le propose dans la position debout).

Chacune de ces possibilités peut correspondre à une situation expressive différente (si l'on est côte à côte avec quelqu'un par exemple, ou si l'on est légèrement éloigné de l'autre personne). Dans l'exercice, c'est la claire conscience de ce qu'il est choisi d'expérimenter qui importe (puis il est possible de choisir l'autre possibilité).

De plus, si l'interlocuteur (ou l'objet) se trouve devant soi, le penchement du torse pourra avoir un aspect révérencieux (Figure 8), ou simplement participer d'un mouvement vers le bas (pour aller chercher ou toucher un objet par exemple), ou d'une position basse (se préparer à courir par exemple). Dans tous les cas, il est l'élément central d'une opposition entre la tête et le bassin (ou les jambes) :

Figure 8 : Penchements du torse vers l'avant dans le genre normal : avec marque de respect/pour saisir un objet/pour se préparer à courir (interprétation par l'image)

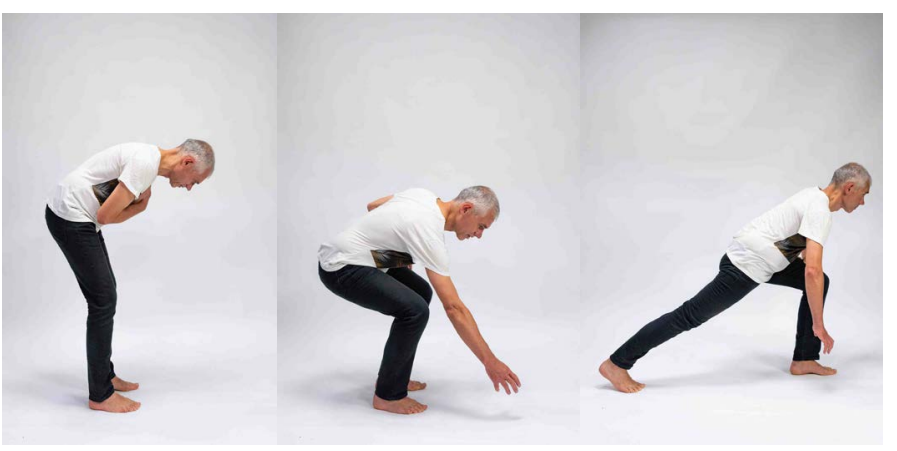

Franck Waille (photographie@David VENIER Université Jean Moulin Lyon 3)

Si nous revenons au texte de 1839 , nous voyons que les indications concernant les trois genres sont directement expressives, celles concernant les six espèces également (lien avec un interlocuteur), mais celles concernant les formes additionnelles (répartition du poids sur l'une ou l'autre hanche) plus du tout : elles sont ici uniquement posturales. C'est dans cette mesure que le travail des attitudes du torse a pu être en grande partie ramené au seul travail de l'équilibre harmonique, comme c'est très clairement le cas chez Stebbins.

\section{—_ Les attitudes du torse et l'équilibre harmonique dans les transmissions américaines}

La notion d'équilibre harmonique (et celle de gymnastique harmonique) a fait débat en 
Amérique du Nord quant à son origine (certains ont remis en cause la paternité de Delsarte concernant ces expression et les pratiques allant avec). J'ai montré comment l'essentiel du manuscrit Harmonic Gymnastics de Mackaye pouvait être mis en lien avec des documents indiquant clairement la paternité delsartienne (cf. Waille, 2017). Ajoutons que si dans la documentation actuellement disponible (qui est très parcellaire rappelons-le, tout spécialement au niveau de la pédagogie du corps et de la voix - cf. Waille, 2015a), les expressions d'équilibre harmonique et de gymnastique harmonique ne se rencontrent pas, elles sont à mettre directement en lien avec deux expressions proches qui, elles, sont identifiables et qui concernent le mouvement expressif, pour lequel Delsarte utilisait un terme venu de son univers de musicien et de chanteur (« harmonique ») : « le geste est harmonique par la multiplicité des parties qui concourent à sa formation » $(1839$, p. 23$)$; et la « loi harmonique du geste » (1858) pour parler de sa grande loi du geste, celle des oppositions (pour la statique comme pour la dynamique). L'adjectif « harmonique » est aussi sous sa plume utilisé dans un cadre très général qui synthétise son approche, comme dans le titre de l'un des feuillets de qui nous est parvenu : Base de l'art. Science des formes. Rapport harmonique du corps et de l'âme (DC, box 1, folder 21 , item 1). Donc, si nous ne rencontrons pas directement les expressions d'équilibre et de gymnastique harmoniques dans les documents de Delsarte aujourd'hui accessibles, cela ne peut exclure que ces expressions ne soient de lui, et qu'il les ai en particulier utilisées lors de ses enseignements pratiques.

Retenons ici que la notion de geste ou de posture harmonique est à mettre en lien avec les différentes parties du corps concourant à l'équilibre ou au mouvement, et que cela est source d'harmonie. Delsarte a repéré l'équilibre harmonique par ses observations du quotidien comme dans la statuaire grecque, à laquelle il faisait sans cesse référence dès qu'il s'agissait des lois de l'harmonie et de l'équilibre'.

4 «II racontait qu'il avait, lui, étudié pendant quinze ans les
Mackaye a transmis la notion d'équilibre harmonique de la manière suivante :

Le centre commun de toutes les formes de mouvement dans le corps est le centre de gravité qui doit normalement être à la verticale des pieds.

L'équilibre de ce centre qui doit coller à la perfection avec la forme physique de leur action expressive est appelé l'équilibre harmonique, ou cet équilibre du corps sur ses pieds qui amène l'ensemble de ses parties à avoir entre elles les meilleures relations possible pour qu'il y ait une précision subtile dans leur coopération harmonique. ${ }^{5}$

II transmet ensuite (1886, leçon 4) deux séries d'exercices « pour la mise en place d'un équilibre harmonique instinctif »[«for the establishment of an instinctively harmonic poise of the body »]. Je propose d'illustrer ainsi (Figure 9) la première série (deux exercices) qu'il dénomme exercices d'« équilibre harmonique pour l'unité » (Harmonic poise for Unity - 1886, p. 6-8) :

Figure 9 : Exercices pour l'équilibre harmonique favorisant l'unité [" for Unity »] (Mackaye, 1886, p. 6-8) (interprétation par l'image)

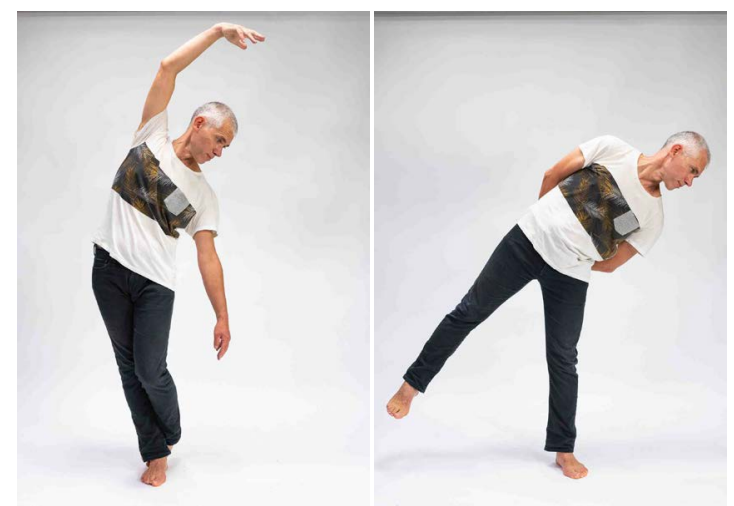

Franck Waille (photographie@David VENIER Université Jean Moulin Lyon 3)

poses des statues antiques $»$ (Arnaud, 1882, p. 198).

5 " The common centre of all forms of motion in the body, is its center of gravity, when normally erect upon its feet. The poise of this centre which most perfectly fits the physical forms for their expressive action, is called the Harmonic Poise. Or that poise of the body upon its feet which brings all its different parts into the best relation for a subtle precision of harmonic cooperation " (Mackaye, 1886, leçon 3, p. 5-6). 
Nous verrons plus bas comment mettre ces exercices en lien avec des notes manuscrites de Delsarte.

Mackaye transmet ensuite une série d'« Exercices dans l'équilibre harmonique pour la sensation d'être un » (Exercises in the harmonic poise for Oneness-1886, p. 8-9). Je propose d'illustrer ainsi le premier exercice (Figure 10) :

Figure 10 : Exercice në 1 pour l'équilibre harmonique favorisant la sensation d'être un [« for Onesness »] (Mackaye, 1886, p. 8) : vers l'arrière et vers l'avant en restant droit (interprétation par l'image)

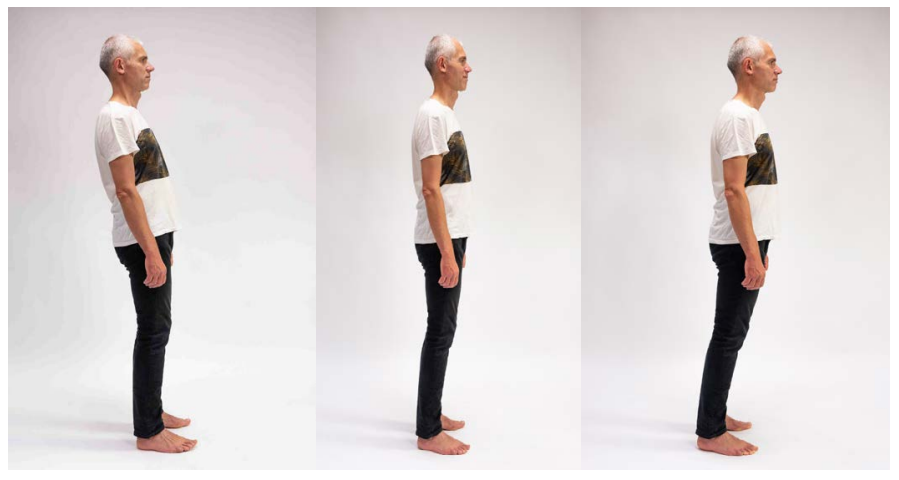

Franck Waille (photographie@David VENIER Université Jean Moulin Lyon 3)

C'est à partir de ce dernier exercice que nous pouvons illustrer la conception différente de l'équilibre harmonique qu'a développé Stebbins par rapport à Mackaye. Mackaye privilégie la sensation d'" être un » associée à la sensation du poids et à son transfert dans le corps ; il met ainsi l'accent sur l'association que fait Delsarte entre le poids et l'unité, entre la sensation de poids et la sensation de soi (cf. Waille, 2016, p. 250-257). Stebbins, elle, privilégie les rapports harmoniques des grandes parties du corps lors de ces transferts de poids. Chez Stebbins en effet, ces deux exercices (Figure 11) deviennent :
Figure 11 : Exercice pour l'équilibre harmonique dans le plan sagittal (\#7 \& 8 dans la transmission de Stebbins, 1977, p. 96-97) : oppositions torse / tête \& bassin lors du transfert du torse vers l'arrière et vers l'avant (interprétation par l'image)
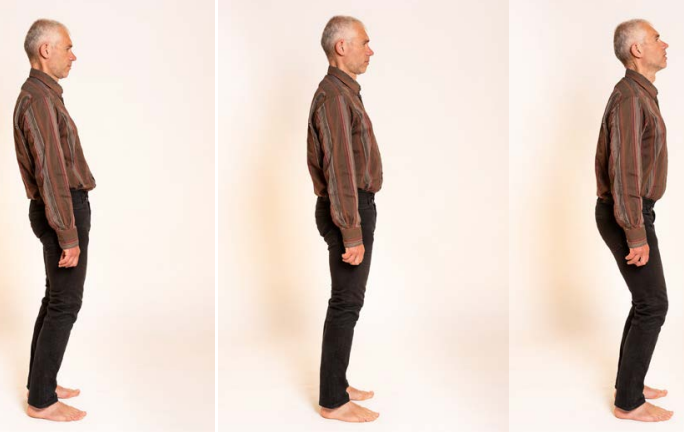

Franck Waille (photographie@David VENIER Université Jean Moulin Lyon 3)

Nous voyons alors que nous retrouvons les trois genres des attitudes du torse décrits par Delsarte en 1839. Dans ce cas, il y a bien moins d'utilisation des muscles pour le maintien, dans la mesure où ce sont les rapports d'opposition entre les éléments de l'axe central du corps qui joue en grande partie ce rôle de maintien. Et la notion d'équilibre se comprend aisément : si dans la version transmise par Mackaye, le poids navigue vers l'avant ou l'arrière du pied, dans l'alternance des trois genres du torse utilisée ici par Stebbins, l'égale répartition du poids sur l'ensemble du pied reste constante, le déplacement du thorax dans une direction étant compensé par celui de la tête et du bassin dans l'autre (comme nous l'avons indiqué pour les trois genres du torse - cf. supra).

Le deuxième exercice « dans l'Équilibre Harmonique pour la sensation d'être un » (Exercises in the harmonic poise for Oneness - 1886, p. 8-9) transmis par Mackaye est une variation du premier, avec les jambes écartées. II est fortement apparenté à l'exercice proposé par Delsarte autour de la huitième attitude des jambes (le transfert progressif du poids sur la jambe qui est avancée ou sur celle qui est reculée - cf. supra), à ceci près que le départ est indiqué avec le poids sur la jambe arrière tendue (Figure 12): 
Figure 12 : Exercice në 2 d'équilibre harmonique favorisant la sensation d'être un ["for Onesness »] jambes écartées (Mackaye, 1886, p. 9) (interprétation par l'image)

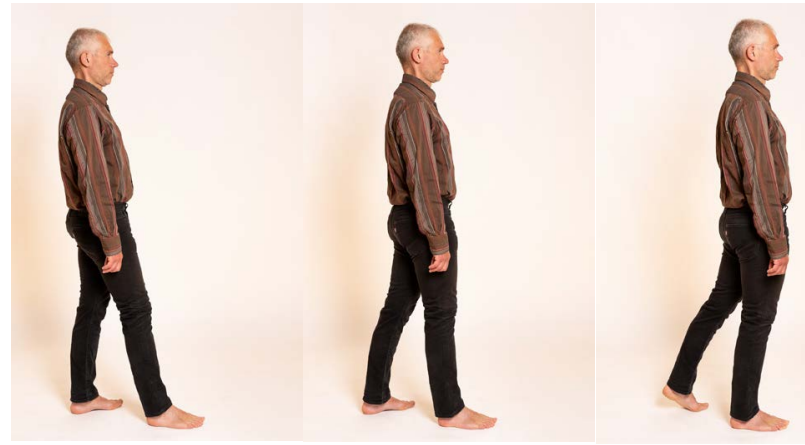

Franck Waille (photographie@David VENIER Université Jean Moulin Lyon 3)

Stebbins propose elle aussi une version avec les jambes écartées ; elle se réfère alors directement à la statuaire antique, ce qui est conforme aux indications de Delsarte et à l'observation de ces statues présentant des oppositions thorax / tête \& bassin (Figure 13) :

Figure 13 : Exercice d'équilibre harmonique dans le plan sagittal jambes écartées (Stebbins, 1977, p. 94) (interprétation par l'image) / Alexandre le Grand (Musée du Louvre, salle du Manège, $\mathrm{n}^{\circ}$ d'entrée LL 11)
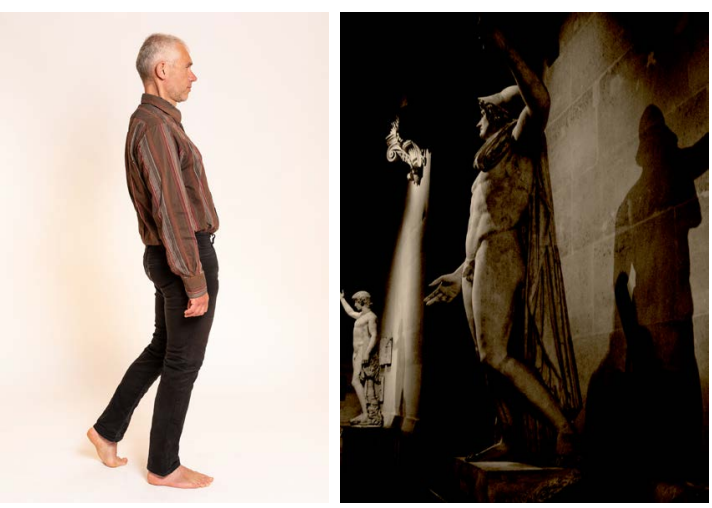

Franck Waille (photographie@David VENIER - Université Jean Moulin Lyon 3) / photographie@ auteur

C'est probablement de cette observation de la statuaire antique que Delsarte a tiré une règle concernant le rapport de sympathie entre la tête et la jambe forte indiquée par Delaumosne, qui écrit que " la tête doit être tournée du côté de la jambe forte » $(1874$, p. 77$)$. Cette règle structure les exercices transmis par Stebbins.

Stebbins, dans le deuxième chapitre de son ouvrage portant sur l'« Équilibre harmonique du maintien » [Harmonic Poise of Bearing] (1977, p. 91-102) utilise une grande partie de la matière du travail du torse. Dans le plan sagittal, comme nous venons de le voir, mais aussi précédemment dans le plan frontal, avec les variations de la répartition du poids sur une jambe et sur l'autre, et en faisant alterner les positions avec l'interlocuteur d'un côté ou de l'autre. Elle sélectionne ainsi une partie des données des espèces du torse (Figure 14), qu'elle a la trouvaille pédagogique de faire travailler de manière liée et répétitive (elle indique de passer de manière fluide d'une position à une autre) :

Figure 14 : Exercices d'équilibre harmonique dans le plan frontal ( $\mathrm{n}^{\circ} 1$ à 3 dans la transmission de Stebbins, 1977, p. 91-93) (interprétation par l'image)

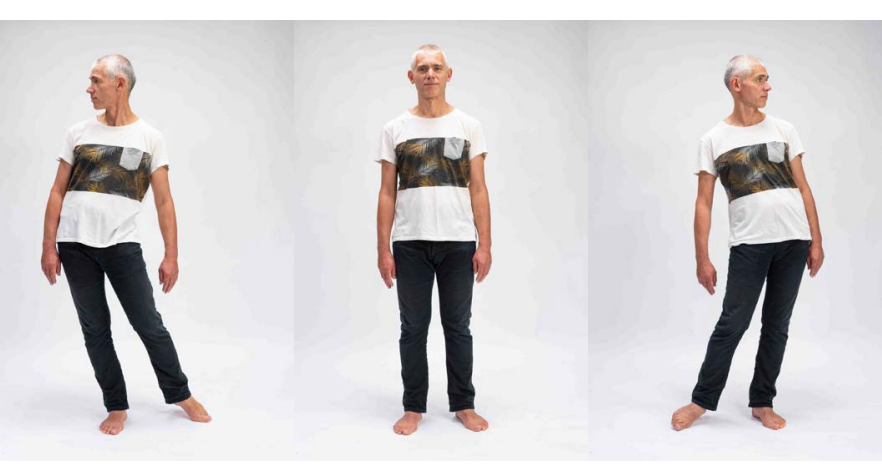

Franck Waille (photographie@David VENIER Université Jean Moulin Lyon 3)

J'avais déjà indiqué dans ma thèse que chez Stebbins, la douzième leçon concernant le torse présente une anomalie majeure par rapport aux leçons se rapportant aux autres parties du corps : s'il y a présentation des attitudes du torse, il n'y a pas d'accord de neuvième qui en soit proposé, et surtout pas d'exercice correspondant. De manière tout à fait incohérente, la section « æsthetic gymnastics » concluant cette courte leçon propose en effet un travail pour les bras (1977, p. 210-211) (alors que les bras ont déjà bénéficié des trois leçons précédentes). Tout le contenu du transfert du poids sur une jambe et sur l'autre est donc passé aux exercices du deuxième chapitre sur l'équilibre harmonique. 
Concernant ce balancement des parties centrales du corps appelé l'équilibre harmonique dans le delsartisme américain (nous verrons plus bas que cette notion d'équilibre harmonique était plus large à l'origine), Delsarte l'a repéré autour de lui. C'est le balancement naturel qui peut s'observer dans la station debout, mais aussi et tout spécialement dans la marche comme le rappelle la delsartiste Henrietta Hovey (qui travailla avec Gustave Delsarte, le fils de François ayant poursuivi la transmission sur Paris des enseignements de son père après la mort de ce dernier). Hovey indique que «l'expression de l'équilibre du corps, dès lors que les pieds sont concernés, doit uniquement être étudié à travers la marche ${ }^{6}$. Nous pouvons découvrir, en étant attentif à nos mouvements quand nous marchons, les rapports torse / tête \& bassin dans tous les plans et dans les rotations (la marche humaine étant controlatérale). Hovey indique que ce qui est appelé l'équilibre harmonique en Amérique du Nord (le « soit disant équilibre harmonique ") est une position par laquelle nous passons quand nous allons d'un bon pas ${ }^{7}$. La marche présente des rapports harmoniques entre la tête, le torse et le bassin dans tous les plans de manière simultanée. Les exercices qui en sont tirés individualisent chaque plan de manière spécifique et successive, étant entendu qu'ils ne sont qu'un temps d'apprentissage.

Delsarte a également repéré ce balancement sur les statues antiques, balancement apparaissant dès Le Doryphore (le Porte lance) du sculpteur grec Polyclète ( $\mathrm{V}^{\mathrm{e}}$ siècle av. J.-C.) qui a servi de modèle esthétique - il est parfois dénommé le Canon (cf. Muller-Dufeu, 2002, p. 397). Le Doryphore « est le premier spécimen du corps masculin à la beauté idéalisée dans l'art grec classique. (...) Sa forme équilibrée et sa position en contrapposto (une jambe fléchie entraîne l'inclinaison des hanches, ce qui donne un mouvement virtuel à la figure) donnent nais-

6 «The expression of a poise of the body should so far as the feet are concerned, be studied through walking only " (TSC, fol$\operatorname{der} 728$, p. 1).

7 « The so called harmonic poise (...) is a pose to which we come or through which we pass in taking a good step » (id., p. 5). sance à la notion occidentale d'équilibre parfait dans le rapport entre les parties du corps et l'ensemble » (Smalls, 2003, p. 266, note 19). C'est une « représentation dynamique du corps à travers le mouvement s'enroulant autour d'un axe central (...) une combinaison de mouvement et de repos, d'équilibre et d'asymétrie : une danse des oppositions » (Barba, Savarese, 2008, p. 170). Ma compréhension de la statuaire grecque classique est que cette statuaire présente un arrêt sur un moment de la marche, comme un cliché photographique gravé dans le marbre : c'est pour cette raison qu'elle contient tous les éléments de ce que nous avons jusqu'ici appelé l'équilibre harmonique.

\section{La notion complète d'équilibre harmonique}

S'il va de soi que les positions du torse, et donc ses rapports avec les jambes et la tête, jouent un rôle central dans l'équilibre harmonique, il est important de relever que chez Delsarte, cet équilibre harmonique intègre ce que je viens de présenter, mais va au delà. Ainsi, dans le compte-rendu des cours de 1839, nous trouvons indiqué :

(...) passons à l'application, et commençons par les lois de l'équilibre. Elles sont bie simples, quoique difficiles à observer.

1 1ë Au penchement du torse correspond l'allongement de la jambe en sens opposé.

2ë Lorsqu'un bras est ajouté à la pesanteur du torse déjà penché, l'autre bras doit se soulever pour former contrepoids.

3 ë Au penchement de la tête, répond encore le penchement opposé du torse.

4 ë En regardant au fond d'un puits, les deux bras peuvent se retirer en arrière si l'on est également supporté par les deux jambes ; de même que les deux bras peuvent venir en avant par suite d'un reculement du torse en arrière. Mais cela 
n'est tolérable qu'en première ou dans une attitude analogue, telle que la deuxième rapprochée ou une petite troisième (Delsarte, 1839, p. 24).

Dans ce texte, « première », « deuxième » et « troisième » désignent les trois premières attitudes des jambes. Chacun des quatre points devait donner lieu à un ou à des exercices spécifiques, qui devraient pouvoir se retrouver dans les exercices transmis par les élèves de Delsarte.

Le premier point, par exemple, inspire directement l'exercice transmis par Mackaye « pour l'unité » [Harmonic poise for Unity], dans lequel le torse est en opposition avec la jambe levée (dans le plan frontal alors). Je l'ai, par ailleurs, appliqué dans les photographies présentant les exercices d'accord de neuvième du torse. L'opposition entre le torse et la jambe est la base des oppositions entre le torse et la hanche, quand le poids repose sur cette jambe et que donc elle ne peut s'écarter (nous en avons vu des exemples dans les attitudes du torse).

Le deuxième point fait penser à l'arabesque en danse académique, arabesque à laquelle je suis arrivé par un simple prolongement d'un exercice de Delsarte transmis par l'un de ses dessins et qui présente la succession d'une série d'oppositions entre différentes parties du corps (tête / main; thorax / tête et bassin; bassin / tête ; thorax / jambe; bras / jambe) (cf. Waille, 2011 , p. $825-826$, \& 2016, p. 422-426) ; il inspire indirectement l'autre des exercices transmis par Mackaye «pour l'unité » (l'ajout du bras au poids du torse n'est pas alors compensé par le soulèvement de l'autre bras, mais c'est le basculement du bassin en opposition qui fait contrepoids).

Le troisième point, quant à lui, est ce qui soustend les rapports d'opposition entre la tête et le thorax dans le travail des attitudes du torse (et cela se retrouve donc également dans le travail des attitudes de la tête qui, sans cela, deviendrait un travail purement mécanique et sans saveur).

Le quatrième point se retrouve dans divers exercices publiés par Giraudet, qui transmet par ailleurs de très nombreux exercices d'opposi- tion pour les différentes parties du corps (tête / épaule ; tête / torse ; bras / tête ; bras / torse etc.) (1895, p. 103-105 + dessins XXIX-XXI).

II est significatif que Delsarte commence bien, à cette époque, ses cours pratiques par la question de l'équilibre du corps, et que cet équilibre est conçu comme la coopération harmonieuse de différents agents : cela est strictement la notion d'équilibre harmonique (même si elle n'est pas nommée ainsi dans ce manuscrit).

II est notable que toutes les indications données ici concernent des rapports d'opposition entre le torse et d'autres parties du corps (jambe, bras, tête). Cela indique clairement que pour Delsarte, dès les débuts de son enseignement, le torse a un rôle central dans le travail des oppositions, et donc de l'équilibre général du corps, autrement dit dans les questions posturales. Et le travail des attitudes du torse, dans ce même manuscrit, suit immédiatement ce passage initial sur l'équilibre.

Mais dans un autre manuscrit, on voit que Delsarte, lors d'une conférence, indique que l'équilibre se travaille aussi avec les autres parties du corps:

Lorsque mon bras s'élève vers ma tête, ma tête fait la moitié du chemin et s'incline. Voilà une opposition de mouvements. Si ma main se détache ainsi ma tête s'élève, et ce petit mouvement de ressort produit par le carpe a son écho dans la tête. II ne se passe pas un mouvement dans la main qui n'ait son écho, sa répercussion dans la tête. (...) $\mathrm{Si}$ je tournais véhémentement la tête comme cela avec un mouvement semblable du bras, je tomberais (1858).

Nous voyons ainsi que la loi des oppositions, pierre angulaire de la statique comme de la dynamique chez Delsarte (cf. Waille, 2016, p. 205-213), est la loi qui régit l'équilibre harmonique dans toutes les situations expressives. Elle concerne tant les rapports des éléments de l'axe central du corps (torse, tête, bassin-jambes) que les rapports entre les autres éléments corporels. C'est par elle que, pour Delsarte, s'établit l'harmonie dans le mouvement, indissociable d'une certaine qualité ou état de corps, qu'il évoque par 
l'expression d'« aisance dans la force » (Mackaye, 1869 / 1870b, p. 91), ou de « force et suavité » (Delsarte, s.d.), ou encore d'«Énergie douce » (ibid.).

\section{Conclusion}

Les nouvelles approches que je présente ici de certaines informations venues de manuscrits ou de publications font écho, d'un point de vue méthodologique, à la mise en garde qui a été faite dans mes publications de 2011 comme de 2016 : au moment où elles sont faites, les photographies sont à la fois ma compréhension des informations recueillies, et une part de mon commentaire de celles-ci. Elles n'ont ni caractère normatif ni prétention esthétique : il ne s'agit pas de donner la bonne manière de faire ces exercices, mais de présenter un outil de communication rendant plus accessible des descriptions souvent difficiles d'accès par le seul biais d'un texte. Si elles cherchent à être aussi fidèles que possible aux sources sur lesquelles elles s'appuient, elles n'en restent pas moins mon interprétation à un moment donné des informations recueillies, d'autant plus qu'elles prennent inévitablement en compte les réalités du corps photographié dans ses aspects anatomiques comme dans son expérience antérieure de travail du mouvement. Elles sont, par nature, susceptibles d'évoluer, et c'est le motif premier de cet article : proposer une mise à jour d'interprétations possibles de documents, mise à jour qui reste elle aussi susceptible d'évoluer. Par ailleurs, la variété des informations transmises par différents élèves et/ou documents renvoie très certainement aux différentes variations et nuances que Delsarte introduisait dans son enseignement, variations qui invitent à cette dynamique ouverte et sans cesse susceptible de nouvelle interprétation. La rencontre avec l'approche de Clóvis Massa a mis en valeur la richesse de cette variété d'interprétations possibles.

Les exercices présentés dans cet article, pour le torse mais aussi pour les jambes (dans l'article précédent), et plus généralement pour tout ce qui relève de l'opposition des différentes parties du corps en vue de l'équilibre et de l'harmonie dans la statique comme dans la dynamique, sont des éléments centraux dans le travail expressif delsartien puisqu'ils participent directement à la construction d'un équilibre harmonieux et harmonique servant de base à l'expression. Pour reprendre les mots de Mackaye, ce travail « tend à développer chez l'individu une obéissance instinctive, inconsciente, sans effort et spontanée aux lois universelles qui développent l'aisance, la précision et l'harmonie du mouvement, et ainsi sublime et donne de la puissance à l'expression humaine $"{ }^{8}$ II s'agit, en définitive, d'un processus de réorganisation motrice basé sur des observations minutieuses des fonctionnements les plus optimaux et naturels. Comprenons « naturels » dans le sens aristotélicien du terme : « (...) la nature ne fait rien de vain, mais réalise toujours le meilleur parmi les possibles qui correspondent à l'essence de chaque animal »(Aristote, 1973, p. 13 [Marche des animaux, 2, 704 b]). Cela trouve un écho en termes de fonctionnements optimaux dans une théorie récente du contrôle du mouvement présentée par Alain Berthoz, qui suggère « que le système moteur réalise les mouvements en suivant le principe de minimum d'énergie » (1997, p. 165). II devient clair que chez Delsarte nous sommes dans un processus de travail en profondeur sur le fonctionnement de la personne qui, pensé initialement dans une optique expressive, comporte une dimension de réorganisation motrice de type thérapeutique. Si celle-ci peut être dissocie de l'optique artistique initiale de Delsarte, il pourrait être intéressant toutefois d'être à l'écoute d'une autre proposition de ce dernier. L'artiste du XIXe siècle suggérait que la dimension expressive de l'être humain est ce qui permet par excellence l'unité de la personne, le lien profond entre le corps et ce qui l'anime, l'âme (cf. Waille, 2011, p. 311-312). II s'agirait alors de

\footnotetext{
8 « (...) a series of exercices have been invented called Harmonic gymnastics which seek[s] to develope an instinctive, unconscious, effortless, and spontaneous obedience, on the part of the individual, to those universal laws which develope ease, precision and harmony of motion, and thus sublimate, and potentize expression of man » (Mackaye, 1886, p. 5).
} 
retourner le point de vue et de s'interroger sur ce qu'est le thérapeutique, et de la viabilité pour celui-ci de se dissocier de la perspective expressive associée à celle d'unité de la personne. Et si, en définitive, l'expressif n'était pas au service du thérapeutique, mais le thérapeutique une dimension de l'expressif ? Ou encore, et si le thérapeutique et l'expressif étaient indissociables ? Peut-être la dimension expressive pourrait-elle être l'horizon le plus enviable pour la personne, celui vers lequel elle serait le plus pleinement elle-même, ou agiraient en synergies toutes ses dimensions, y compris sa dimension spirituelle? II me semble que c'est cela que nous suggère, en ce début de $X X I^{e}$ siècle, l'artiste et pédagogue du XIX ${ }^{\mathrm{e}}$ siècle.

\section{Abrviations}

DC [= Delsarte Collection] : " François Alexandre Nicolas Delsarte Papers, Mss. 1301, Louisiana and Lower Mississippi Valley Collections, LSU Libraries, Baton Rouge, La. ».

$\mathrm{FH}$ : Fonds Hamel, Fonds des archives du Séminaire de Québec : SME (1626-1994), Musée de la civilisation, Service des collections, des archives historiques et de la bibliothèque, Québec (Québec), Canada.

MPF : The papers of the Mackaye Family, Baker Library, Special Collections, Dartmouth College, Hanover, New Hampshire, USA (Manuscript ML-5, Series 7, Steele MacKaye, 18481916, box 7 \& box 8 / Manuscript ML-5, Series 22, Addendum 1, box 219).

PRH : The Papers of Richard Hovey, Baker Library, Special collections, Dartmouth College, Hanover, New Hampshire, USA.

TSC : Ted Shawn Collection, Lincoln Center, Jerome Robbins Dance Division, The New York Public Library for the Performing Arts, New York, USA, cote : (S) *MGZMC-Res. 31, série VIII « Delsarte System of Expression».

\section{Bibliographie et sources (imprimes ou manuscrites)}

ALEXANDER, Frederick Mathias. L'usage de soi. Bruxelles : Éditions Contredanse [The Use of the Self], 1996 [1923].

ARISTOTE. Marche des animaux. Mouvements des animaux. Index des traités biologiques. Paris : Les Belles Lettres, 1973.

ARNAUD, Angélique. François del Sarte, ses découvertes en esthétique, sa science, sa méthode, précédé de détails sur sa vie, sa famille, ses relations, son caractère. Paris: Ch. Delagrave, 1882.

BAINBRIDGE-COHEN, Bonnie. Sentir, ressentir et agir. L'anatomie expérimentale du Body-Mind Centering ${ }^{\circledR}$ (traduction de Sensing, feeling and action, par Madie Boucon). Bruxelles: Nouvelles de Danse në 50, 2002.

BARBA, Eugenio. SAVARESE, Nicola. L'Énergie qui danse. Dictionnaire d'anthropologie théâtrale, Montpellier : L'Entretemps, 2008.

BARTHÉLÉMY, Yva. La voix libérée. Paris : Robert Laffont, 1984.

BERTHOZ, Alain. Le sens du mouvement. Paris : Odile Jacob, 1997.

BERTHOZ, Alain. La décision. Paris : Odile Jacob, 2003.

CABLE (s.d.), Notebook of Cable while studying with Delsarte [cahier de Cable étudiant avec Delsarte] (DC, box 2c, folder 132).

CHEVALIER, Jean; GHEERBRANT, Alain. Dictionnaire des symboles. Paris : Bouquins, Robert Laffont / Jupiter, 1982. 
DAWN CLARK, Janis. The Influence of the Delsarte System of Expression on American Acting 1871-1970. Ph.D. Dissertation, Washington State University, 1982.

DELAUMOSNE, Abbé. Pratique de l'art oratoire de Delsarte. Paris : Joseph Albanel, 1874.

DELSARTE, François. École de Delsarte, École de chant morale et scientifique. Notes et compte-rendu de ses cours, 1839 (DC, box 11b, folder sans \#). Différents transcripteurs, avec annotations et signature de Delsarte, 1839.

DELSARTE, François. Cours de M. Delsarte aux Sociétés savantes, cours në 8 (DC, box 12 b, folder 54), 1858.

DELSARTE, François. Esthétique appliquée, cours de F. Delsarte. Exposition en neuf leçons de l'art de l'orateur, du peintre et du musicien. Offert à $M$. Delsarte par son élève Alphonse Pages (DC, box 12c, folder 40), 1859.

DELSARTE, François. Beau (DC, box 9-10, folder OS 36a, document 3), (s.d.).

FERNANDES, Ciane. The Moving Researcher: Laban/Bartenieff Movement Analysis in Performing Arts Education and Creative Arts Therapies. Kingsley : Jessica Publisher, 2014.

GIRAUDET, Alfred. Mimique, Physionomie et Gestes. Méthode Pratique D'après le système de François Del Sarte pour servir à l'expression des sentiments. Paris : Ancienne Maison Quantin / Librairies-Imprimeries Réunies, 1895.

GODARD, Hubert. "Le geste et sa perception." In : GINOT, Isabelle; MICHEL, Marcelle. (Org.). La Danse au XXe Siècle. Paris : Bordas, 1995, p. 224-229.

GUIDO, Laurent. L’Âge du rythme - Cinéma, musicalité et culture du corps dans les théories françaises des années 1910-1930. Lausanne : Éditions Payot / Jacques Scherrer éditeur, 2007.
HAMEL, Thomas-Étienne. Cours d'éloquence parlée d'après Delsarte. Québec : Imprimerie de la compagnie de l'Événement, 1906.

JOUSSE, Marcel. Étude de psychologie linguistique. Le style orale rythmique et mnémotechnique chez les verbomoteurs. Paris, Beauchesne, 1925.

LABAN, Rudolf. La maîtrise du mouvement. Arles : Actes sud, 1997.

MACKAYE, James Steele. Notebook of Mackaye while studying with Delsarte \#3 / 7 [cahier de Mackaye étudiant avec Delsarte në 3/7] (DC, box 12b, folder 7), 1869 / 1870a.

MACKAYE, James Steele. Notebook of Mackaye while studying with Delsarte \#12 [cahier de Mackaye étudiant avec Delsarte në 12] (DC, range 35), 1869 / 1870b.

MACKAYE, James Steele. Harmonic Gymnastics (DC, box 6, folder 76), 1886.

MASSA, Clóvis Dias. A significação corporal a partir de François Delsarte. Projeto de Graduação, ligado à FAPERGS através da pesquisa teórica "Emprego de Signos Gestuais", Departamento de Arte Dramática da Universidade Federal do Rio Grande do Sul (UFRGS). Porto Alegre, 1991.

PORTE, Alain. François Delsarte, une anthologie. Paris, IPMC, 1992.

PRADIER, Jean-Marie. La scène et la fabrique des corps. Éthnoscénologie du spectacle vivant en Occident. Ve siècle avant Jésus-Christ-XVIII siècle. Talence : Presses Universitaires de Bordeaux, 2000.

RUYTER, Nancy Lee Chalfa. "Delsarte, son système et les États-Unis », in François Delsarte, 1811-1871. Sources-Pensée. ChâteauvalIon, Théâtre National de la Danse et de I'Image, p. 33-37, 1991. 
SMALLS, James. L'homosexualité dans l'art. New York : Parkstone Press, 2003.

STEBBINS, Genevieve. Delsarte System of Expression. New York, Dance Horizons (réédition

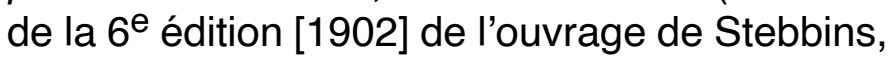
initialement intitulé Delsarte System of Dramatic Expression, publié pour la première fois en 1885), 1977.

VAN HYMBECK, Auguste. Résumé des leçons de chant, grammaire, d'attitudes etc. du cours de $M$. Delsarte. Cahier de Van Hymbeck étudiant avec Delsarte (DC, box 8, folder 9, document 2), 1843.

WAILLE, Franck. Corps, arts et spiritualité chez François Delsarte (1811-1871). Des interactions dynamiques. Lille, ANRT, (version de soutenance [2009] en ligne, chapitre par chapitre, à l'adresse https://scd-resnum.univ-lyon3.fr/out/ theses/2009_out_waille_f.pdf), 2011.

WAILLE, Franck. « Les archives de François Delsarte, sources et produit d'un travail scientifique », in Waille, Damour (2015), p. 19-22, 2015a.

WAILLE, Franck. «Retrouver la vitalité des enseignements de Delsarte : expressifs (ni stéréotypés, ni physiognomoniques) et inscrits dans le mouvement (non figés) », in Waille, Damour (2015), p. 77-94 . 2015b.

WAILLE, Franck. « Alfred Giraudet, artiste lyrique et pédagogue delsartien ", in Waille, Damour (2015), p. 113-126, 2015c.

WAILLE, Franck. " Le travail corporel expressif de Delsarte : des observations du quotidien à un entraînement corporel structuré ", in Waille, Damour (2015), p. 273-280, 2015d.

WAILLE, Franck. La méthode somatique expressive de François Delsarte. Historique, esthétique, anthropologique : de la neurophysiologique à la métaphysique. Montpellier : L'Entretemps, 2016.
WAILLE, Franck. "François Delsarte et les arts de la scène : l'enquête américaine ». Mis en ligne sur le site de l'Acfas (Association francophone pour le savoir), juin 2017 (http://www. acfas.ca/publications/decouvrir/2017/06/francois-delsarte-arts-scene-enquete-americaine) . 2017.

WAILLE, Franck, DAMOUR, Christophe (dir.) François Delsarte, une recherche sans fin. $\mathrm{Pa}-$ ris : L'Harmattan, 2015.

WINKIN, Yves. La Nouvelle Communication. Paris : Seuil, 2000.

Recebido: 26/12/2018 Aprovado: 06/07/2019 